\title{
Responsible research publication: international standards for authors
}

A position statement developed at the $2^{\text {nd }}$ World Conference on Research Integrity, Singapore, July 22-24, 2010

Elizabeth Wager \& Sabine Kleinert

Contact details: liz@sideview.demon.co.uk sabine.kleinert@lancet.com

- Summary

- The research being reported should have been conducted in an ethical and responsible manner and should comply with all relevant legislation.

- Researchers should present their results clearly, honestly, and without fabrication, falsification or inappropriate data manipulation.

- Researchers should strive to describe their methods clearly and unambiguously so that their findings can be confirmed by others.

- Researchers should adhere to publication requirements that submitted work is original, is not plagiarised, and has not been published elsewhere.

- Authors should take collective responsibility for submitted and published work.

- The authorship of research publications should accurately reflect individuals' contributions to the work and its reporting.

- Funding sources and relevant conflicts of interest should be disclosed.

Cite this as:

Wager E \& Kleinert S (2011) Responsible research publication: international standards for authors. A position statement developed at the $2{ }^{\text {nd }}$ World Conference on Research Integrity, Singapore, July 22-24, 2010. Chapter 50 in: Mayer T \& Steneck N (eds) Promoting Research Integrity in a Global Environment. Imperial College Press / World Scientific Publishing, Singapore (pp 309-16). (ISBN 978-981-4340-97-7) 


\section{Introduction}

Publication is the final stage of research and therefore a responsibility for all researchers. Scholarly publications are expected to provide a detailed and permanent record of research. Because publications form the basis for both new research and the application of findings, they can affect not only the research community but also, indirectly, society at large. Researchers therefore have a responsibility to ensure that their publications are honest, clear, accurate, complete and balanced, and should avoid misleading, selective or ambiguous reporting. Journal editors also have responsibilities for ensuring the integrity of the research literature and these are set out in companion guidelines.

This document aims to establish international standards for authors of scholarly research publications and to describe responsible research reporting practice. We hope these standards will be endorsed by research institutions, funders, and professional societies; promoted by editors and publishers; and will aid in research integrity training.

\section{Responsible research publication}

\section{Soundness and reliability}

1.1 The research being reported should have been conducted in an ethical and responsible manner and follow all relevant legislation. [See also the Singapore Statement on Research Integrity, www.singaporestatement.org]

1.2 The research being reported should be sound and carefully executed.

1.3 Researchers should use appropriate methods of data analysis and display (and, if needed, seek and follow specialist advice on this).

1.4 Authors should take collective responsibility for their work and for the content of their publications. Researchers should check their publications carefully at all stages to ensure methods and findings are reported accurately. Authors should carefully check calculations, data presentations, typescripts/submissions and proofs.

\section{Honesty}

2.1 Researchers should present their results honestly and without fabrication, falsification or inappropriate data manipulation. Research images (e.g. micrographs, $\mathrm{X}$-rays, pictures of electrophoresis gels) should not be modified in a misleading way.

2.2 Researchers should strive to describe their methods and to present their findings clearly and unambiguously. Researchers should follow applicable reporting guidelines. Publications should provide sufficient detail to permit experiments to be repeated by other researchers.

2.3 Reports of research should be complete. They should not omit inconvenient, inconsistent or inexplicable findings or results that do not support the authors' or sponsors' hypothesis or interpretation.

2.4 Research funders and sponsors should not be able to veto publication of findings that do not favour their product or position. Researchers should not enter 
agreements that permit the research sponsor to veto or control the publication of the findings (unless there are exceptional circumstances, such as research classified by governments because of security implications).

2.5 Authors should alert the editor promptly if they discover an error in any submitted, accepted or published work. Authors should cooperate with editors in issuing corrections or retractions when required.

2.6 Authors should represent the work of others accurately in citations and quotations.

2.7 Authors should not copy references from other publications if they have not read the cited work.

\section{Balance}

3.1 New findings should be presented in the context of previous research. The work of others should be fairly represented. Scholarly reviews and syntheses of existing research should be complete, balanced, and should include findings regardless of whether they support the hypothesis or interpretation being proposed. Editorials or opinion pieces presenting a single viewpoint or argument should be clearly distinguished from scholarly reviews.

3.2 Study limitations should be addressed in publications.

\section{$4 \quad$ Originality}

4.1 Authors should adhere to publication requirements that submitted work is original and has not been published elsewhere in any language. Work should not be submitted concurrently to more than one publication unless the editors have agreed to co-publication. If articles are co-published this fact should be made clear to readers.

4.2 Applicable copyright laws and conventions should be followed. Copyright material (e.g. tables, figures or extensive quotations) should be reproduced only with appropriate permission and acknowledgement.

4.3 Relevant previous work and publications, both by other researchers and the authors' own, should be properly acknowledged and referenced. The primary literature should be cited where possible.

4.4 Data, text, figures or ideas originated by other researchers should be properly acknowledged and should not be presented as if they were the authors' own. Original wording taken directly from publications by other researchers should appear in quotation marks with the appropriate citations.

4.5 Authors should inform editors if findings have been published previously or if multiple reports or multiple analyses of a single data set are under consideration for publication elsewhere. Authors should provide copies of related publications or work submitted to other journals.

4.6 Multiple publications arising from a single research project should be clearly identified as such and the primary publication should be referenced. Translations and 
adaptations for different audiences should be clearly identified as such, should acknowledge the original source, and should respect relevant copyright conventions and permission requirements. If in doubt, authors should seek permission from the original publisher before republishing any work.

\section{Transparency}

5.1 All sources of research funding, including direct and indirect financial support, supply of equipment or materials, and other support (such as specialist statistical or writing assistance) should be disclosed.

5.2 Authors should disclose the role of the research funder(s) or sponsor (if any) in the research design, execution, analysis, interpretation and reporting.

5.3 Authors should disclose relevant financial and non-financial interests and relationships that might be considered likely to affect the interpretation of their findings or which editors, reviewers or readers might reasonably wish to know. This includes any relationship to the journal, for example if editors publish their own research in their own journal. In addition, authors should follow journal and institutional requirements for disclosing competing interests.

\section{Appropriate authorship and acknowledgement}

6.1 The research literature serves as a record not only of what has been discovered but also of who made the discovery. The authorship of research publications should therefore accurately reflect individuals' contributions to the work and its reporting.

6.2 In cases where major contributors are listed as authors while those who made less substantial, or purely technical, contributions to the research or to the publication are listed in an acknowledgement section, the criteria for authorship and acknowledgement should be agreed at the start of the project. Ideally, authorship criteria within a particular field should be agreed, published and consistently applied by research institutions, professional and academic societies, and funders. While journal editors should publish and promote accepted authorship criteria appropriate to their field, they cannot be expected to adjudicate in authorship disputes.

Responsibility for the correct attribution of authorship lies with authors themselves working under the guidance of their institution. Research institutions should promote and uphold fair and accepted standards of authorship and acknowledgement. When required, institutions should adjudicate in authorship disputes and should ensure that due process is followed.

6.3 Researchers should ensure that only those individuals who meet authorship criteria (i.e. made a substantial contribution to the work) are rewarded with authorship and that deserving authors are not omitted. Institutions and journal editors should encourage practices that prevent guest, gift, and ghost authorship.

Note:

- guest authors are those who do not meet accepted authorship criteria but are listed because of their seniority, reputation or supposed influence

- gift authors are those who do not meet accepted authorship criteria but are listed as a personal favour or in return for payment

- ghost authors are those who meet authorship criteria but are not listed 
6.4 All authors should agree to be listed and should approve the submitted and accepted versions of the publication. Any change to the author list should be approved by all authors including any who have been removed from the list. The corresponding author should act as a point of contact between the editor and the other authors and should keep co-authors informed and involve them in major decisions about the publication (e.g. responding to reviewers' comments).

6.5 Authors should not use acknowledgements misleadingly to imply a contribution or endorsement by individuals who have not, in fact, been involved with the work or given an endorsement.

\section{$7 \quad$ Accountability and responsibility}

7.1 All authors should have read and be familiar with the reported work and should ensure that publications follow the principles set out in these guidelines. In most cases, authors will be expected to take joint responsibility for the integrity of the research and its reporting. However, if authors take responsibility only for certain aspects of the research and its reporting, this should be specified in the publication.

7.2 Authors should work with the editor or publisher to correct their work promptly if errors or omissions are discovered after publication.

7.3 Authors should abide by relevant conventions, requirements, and regulations to make materials, reagents, software or datasets available to other researchers who request them. Researchers, institutions, and funders should have clear policies for handling such requests. Authors must also follow relevant journal standards. While proper acknowledgement is expected, researchers should not demand authorship as a condition for sharing materials.

7.4 Authors should respond appropriately to post-publication comments and published correspondence. They should attempt to answer correspondents' questions and supply clarification or additional details where needed.

\section{Adherence to peer review and publication conventions}

8.1 Authors should follow publishers' requirements that work is not submitted to more than one publication for consideration at the same time.

8.2 Authors should inform the editor if they withdraw their work from review, or choose not to respond to reviewer comments after receiving a conditional acceptance.

8.3 Authors should respond to reviewers' comments in a professional and timely manner.

8.4 Authors should respect publishers' requests for press embargos and should not generally allow their findings to be reported in the press if they have been accepted for publication (but not yet published) in a scholarly publication. Authors and their institutions should liaise and cooperate with publishers to coordinate media activity (e.g. press releases and press conferences) around publication. Press releases should accurately reflect the work and should not include statements that go further than the research findings. 


\section{Responsible reporting of research involving humans or animals}

9.1 Appropriate approval, licensing or registration should be obtained before the research begins and details should be provided in the report (e.g. Institutional Review Board, Research Ethics Committee approval, national licensing authorities for the use of animals).

9.2 If requested by editors, authors should supply evidence that reported research received the appropriate approval and was carried out ethically (e.g. copies of approvals, licences, participant consent forms).

9.3 Researchers should not generally publish or share identifiable individual data collected in the course of research without specific consent from the individual (or their representative). Researchers should remember that many scholarly journals are now freely available on the internet, and should therefore be mindful of the risk of causing danger or upset to unintended readers (e.g. research participants or their families who recognise themselves from case studies, descriptions, images or pedigrees).

9.4 The appropriate statistical analyses should be determined at the start of the study and a data analysis plan for the prespecified outcomes should be prepared and followed. Secondary or post hoc analyses should be distinguished from primary analyses and those set out in the data analysis plan.

9.5 Researchers should publish all meaningful research results that might contribute to understanding. In particular, there is an ethical responsibility to publish the findings of all clinical trials. The publication of unsuccessful studies or experiments that reject a hypothesis may help prevent others from wasting time and resources on similar projects. If findings from small studies and those that fail to reach statistically significant results can be combined to produce more useful information (e.g. by meta-analysis) then such findings should be published.

9.6 Authors should supply research protocols to journal editors if requested (e.g. for clinical trials) so that reviewers and editors can compare the research report to the protocol to check that it was carried out as planned and that no relevant details have been omitted. Researchers should follow relevant requirements for clinical trial registration and should include the trial registration number in all publications arising from the trial. 